\title{
Direct Imaging of Dopant-Elements Distributions in High-Strength Nanostructured Materials by Atomic-Resolution Z-Contrast STEM
}

\author{
Eiji Abe
}

Materials Engineering Laboratory, National Institute for Materials Science, 1-2-1 Sengen, Tsukuba 305-0047, Japan

abe.eiji@nims.go.jp

It is well known that strength of light-weight structural materials such as Al- and Mg-base alloys can be improved by a small amount of additional elements, which may form clusters and/or precipitates at nanometer scale. Quantitative determination of these tiny structures, including how dopant elements distribute within them, is a key issue in this field, though; precise measurements of local compositions at atomic-resolution have been quite difficult because of the limitation of conventional high-resolution electron microscopy. To reveal significant insights into the veiled local chemistry at atomic scale, we here use high-angle annular dark-field scanning transmission electron microscopy (HAADF-STEM) that provides significantly atomic-number sensitive contrast (Z-contrast).

Figure 1 shows Z-contrast images of a platelet precipitate (T1 phase) formed in an Al-5.1 Li-1.9Cu-0.3 Mg-0.2Ag(at\%) alloy. It is obvious that the T1 structure is constructed by three atomic layers differing in compositions; a careful analysis of the intensity profiles leads to an improved structural model (Fig. 1, right). We note that even a few Ag atoms segregated at the matrix/precipitate interfaces are also detected, providing direct evidence that Ag plays a critical role in stabilizing nano-scale precipitates. Figure 2 shows novel types of Mg-base long-period ordered structures, one of which was firstly discovered in a nanocrystalline Mg-1at\%Zn-2at\%Y alloy [1]. Unique chemical orders clearly emerge in Z-contrast images, while such feature may never be deduced from the conventional high-resolution imaging (Fig. 2, left). We further find short-range ordering or clustering of $\mathrm{Y}$ atoms within bright atomic layers in Z-contrast images.

[1] E. Abe et al., Acta Materialia 50 (2002) 3845.

[2] The author would like to thank to the collaborators; T. Honma, K. Hono and T. J. Konno for the Al alloys, T. Itoi, Y. Kawamura for the Mg alloys. 

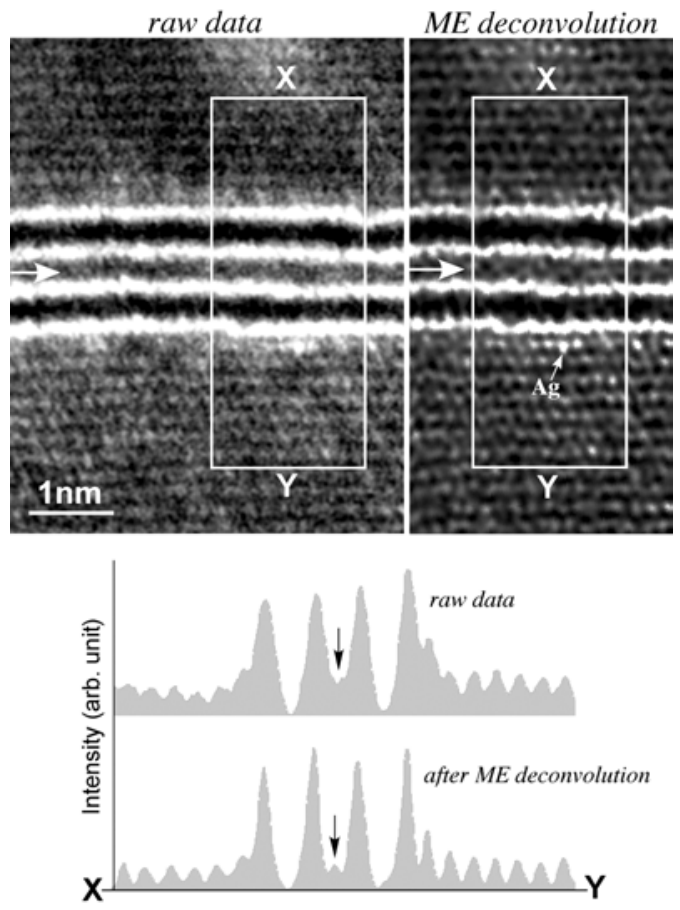

Structure model of T1 phase

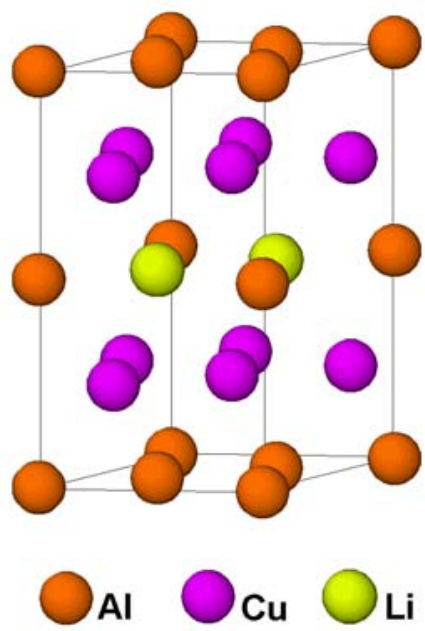

Fig. 1 Structure analysis of the T1-phase precipitated in an Al-Li-Cu-Ag-Mg alloy. Shown left are Z-contrast image and its maximum-entropy deconvolution processed map.
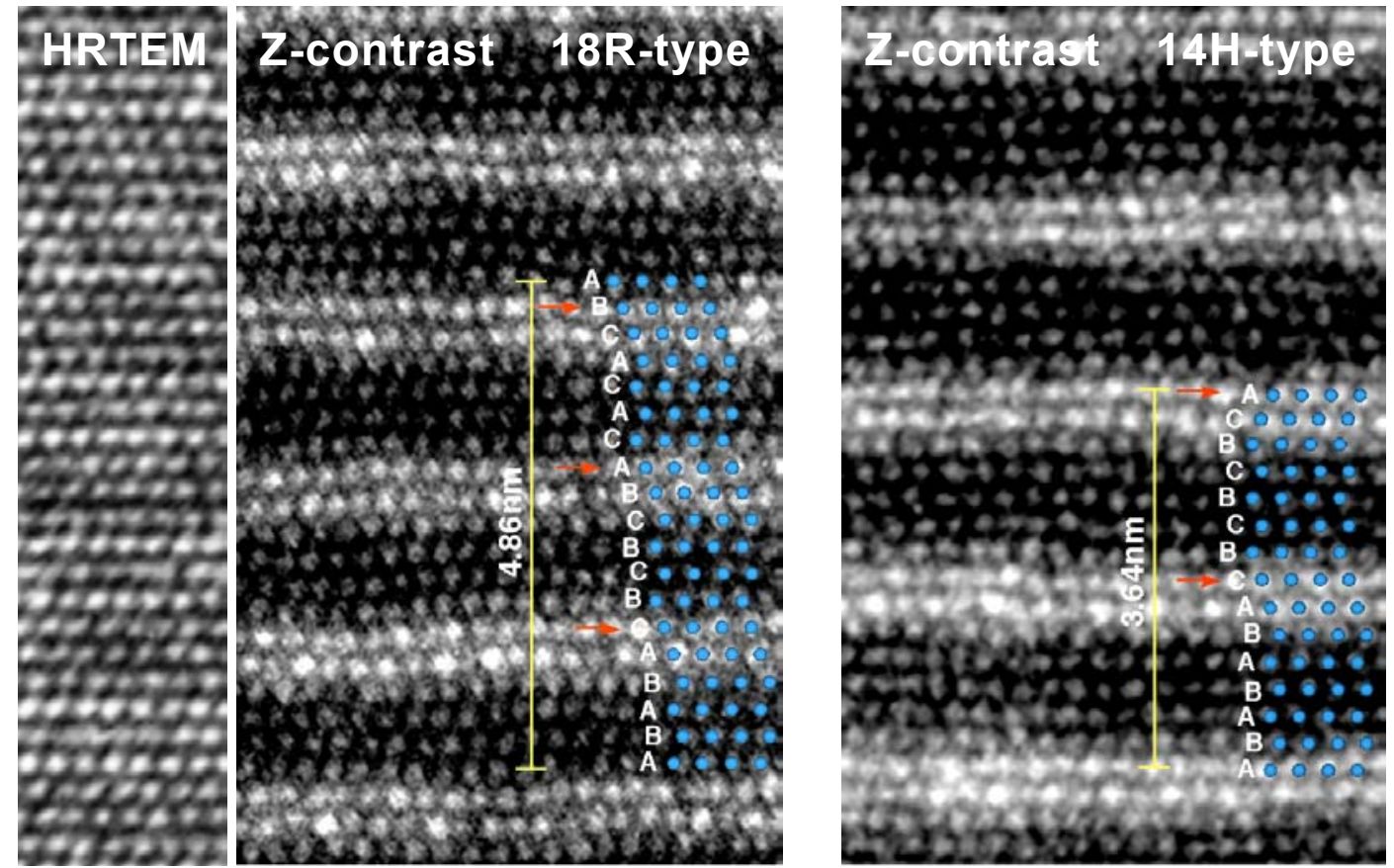

Fig. 2 Atomic-resolution images of novel types of long-period ordered structures formed in Mg-1.0Zn-2.0Y(at\%) alloys. 